\title{
ECOFISIOLOGIA DEL CULTIVO DE MANDIOCA CONSOCIADO CON ALBAHACA Y CORIANDRO EN EL SUBTROPICO DE ARGENTINA
}

\author{
Angela M. Burgos; Pedro J. CENóz y José L. KuszT A(1)
}

\begin{abstract}
RESUMEN: La mandioca es un cultivo que admite consociarse con diversas especies. El objetivo de este trabajo fue evaluar la factibilidad de su consociación con dos cultivos condimentarios, albahaca y coriandro, comparando los componentes del rendimiento de las tres especies bajo el sistema consociado versus sus respectivos monocultivos. El ensayo se realizó en Corrientes, Argentina. Los tratamientos consistieron en tres monocultivos (T2: mandioca, T3: albahaca, T4: coriandro) y un consociado (T1), dispuestos en un diseño de bloques completos al azar con tres repeticiones. Los resultados se analizaron estadísticamente a través de la Prueba de $t$ con muestras no pareadas $(0,05 \%)$. Los resultados mostraron que en el $\mathrm{T} 1$, los componentes del rendimiento del cultivo de mandioca sufrieron reducciones significativas en relación al T2, hubo un incremento significativo del rendimiento del coriandro mediado por el mayor peso de granos por planta en relación al T4, y finalmente el peso fresco y seco de plantas de albahaca, y su partición entre órganos resultó indiferente a los tratamientos. El índice de uso eficiente de la tierra (UET) calculado para este policultivo fue de 3 , lo que demuestra la factibilidad del sistema, permitiendo diversificar la producción para una agricultura sostenible.
\end{abstract}

ABSTRACI: Cassava is allowed to be consociated with various species. The aim of this study was to evaluate the feasibility of consociation cassava crop with two crops species for seasoning, basil and coriander, comparing the yield components of the three species grown under the consociated system comparing with their respective monocultures. The trial was conducted in Corrientes, Argentina. The four treatments consisted of three monocultures (T2: cassava, T3: basil, T4: coriander) and a consociated (T1). The experimental design was randomized complete block with three replicates per treatment and the results were statistically analyzed using the $t$ test with unpaired samples $(0.05 \%)$. The results showed that in $\mathrm{T} 1$, cassava yield components suffered significant reductions respect to $\mathrm{T} 2$, there was a significant increase in coriander yield mediated by the greater weight of the grains per plant compared to $\mathrm{T} 4$, and finally fresh and dry weight of basil plants, and its partition between organs was indifferent to treatments. The rate of the efficient use of land (UET) calculated for this polyculture was 3 , which demonstrates the feasibility of the system, allowing diversify production for a sustainable agriculture.

Palabras claves:Intercultivo, Manihot esculenta Crantz, Ocimum basilicum L, Coriandrum sativum $\mathrm{L}$ Key words: Intercropping, Manihot esculenta Crantz, Ocimum basilicum L, Coriandrum sativum L

\section{INTRODUCCIÓN}

El noreste de Argentina (NEA), concentra más de 20.000 pequeños agricultores que cultivan superficies reducidas en forma permanente; en muchos casos, desde hace más de 150 años. Un factor común en todos ellos, es el elevado nivel de deterioro de los suelos, lo cual ocasiona bajos rendimientos en los principales cultivos de renta. El gran desafío es transformar la agricultura convencional, con énfasis en monocultivo, agresiva hacia el medio ambiente, en una agricultura diversificada, compatible con el medio ambiente y rentable para la familia rural (Ligier, 1997).

(1) Cátedra de Cultivos III, Departamento de Producción Vegetal, Facultad de Ciencias Agrarias (FCA), (UNNE). Sgto. Cabral 2131, (3400) Corrientes. E-mails: burgosangela@agr.unne.edu.ar, burgosangela@hotmail.com 
El uso de los cultivos asociados se presenta como una alternativa válida, que permitiría la mayor diversificación de la producción, el uso más eficiente de la tierra y del tiempo disponible, la reducción de la competencia de malezas y una mayor estabilidad de producción (Cenóz y Ferrero, 2002).

La mandioca (Manihot esculenta Crantz) es un cultivo de origen americano cuyas raíces tuberosas constituyen un alimento básico en la dieta de más de 1000 millones de personas de América, Asia y África fundamentalmente (Alves de Mendoḉa et al.; 2003).

La producción de mandioca en Argentina, se hace en condiciones de monocultivo que tiende a incrementar la erosión de los suelos. Asimismo, es un cultivo multipropósito de ciclo largo, anual ( 9 meses) o bianual ( 21 meses), cuyo amplio marco tradicional de plantación $(1 \times 1 \mathrm{~m})$ permite la consociación con otras especies. El período particularmente apto para consociarlo con otros cultivos resultan los cuatro primeros meses (septiembre-enero) en función a su lento crecimiento inicial; tanto como al finalizar el ciclo productivo anual (abril-junio) cuando alcanza la fase de reposo asociada a la caída de hojas. De esta manera, haciendo un uso más eficiente de los recursos, el espacio agrícola se podría aprovechar intercalando especies y diseñando arreglos espaciales que permitan obtener otros cultivos adicionales (Mojena y Bertolí, 2004). Bajo estas condiciones, aproximadamente el $50 \%$ de las plantaciones de mandioca en Latinoamérica y Africa son actualmente asociadas con otras especies (Alves, 2002).

En la zona del NEA, se efectuaron experiencias de cultivos asociados de mandioca-caupí, mandioca-batata y mandioca-maíz; habiéndose obtenido resultados satisfactorios, con rendimientos que superaron al monocultivo y con beneficios económicos importantes (Cenóz et al., 1995, 1998, 2010; Cenóz y Ferrero, 2002).

En este trabajo la mandioca se consideró como cultivo principal, al que se asociaron dos especies condimentarias seleccionadas por sus ciclos de crecimiento estival en el caso de la albahaca (Ocimum basilicum L.) e inverno-primaveral para el cultivo de coriandro (Coriandrum sativum $\mathrm{L}$ ).

En relación a los cultivos asociados, la albahaca es una especie aromática, que necesita climas templados cálidos, no resiste heladas, exige suelos de mediana fertilidad, livianos y permeables. El rendimiento en Corrientes, Argentina, presenta valores superiores a $12.000 \mathrm{~kg} \cdot \mathrm{ha}^{-1}$ de planta fresca, que se elevan a $18.000 \mathrm{~kg} \cdot \mathrm{ha}^{-1}$ mediante fertilización nitrogenada. Por lo expresado, el potencial de producción sería una alternativa altamente favorable a nivel local (Cenóz et al., 2005).

El coriandro junto con la manzanilla y el orégano, constituyen la principal especie aromática de exportación Argentina (De la Fuente et al.; 2006), pero falta potenciar los rendimientos y la calidad del producto en base a la introducción y evaluación de nuevo germoplasma y su respuesta en distintas regiones del país. Actualmente el cultivo de coriandro no se encuentra difundido en la zona NEA, y tampoco se han encontrado estudios experimentales del comportamiento del mismo bajo las condiciones del bioambiente de Corrientes.

En relación al sistema consociado de especies, ensayos llevados a cabo en India, incorporaron al cultivo de coriandro intersembrado con maíz (Zea maiz L), en los que se encontraron valores de uso eficiente de la tierra (UET) del orden de 1,42 respecto de los monocultivos (Thayamini y Brintha, 2010). Asimismo, evaluaciones de la consociación 
coriandro-berenjena (Solanum melongena L), encontraron no solo efectos positivos en el UET sino también en el control biológico de insectos; y finalmente en consociación con repollo (Brassica oleracea D.C) el UET alcanzó 1,92 (Resende et al., 2010).

Numerosos trabajos publicados demuestran la factibilidad de consociar la mandioca con especies hortícolas (Mattos y Souza, 2005, Hernández et al., 1999; Cenóz et al., 2010); sin embargo no se ha encontrado información científica de la consociación con cultivos de especies condimentarias o "specialities", por lo que este ensayo constituye un primer paso hacia la búsqueda de un sistema de producción agrícola diversificado y alternativo, evaluado desde una perspectiva ecofisiológica que aportará nueva información bajo condiciones subtropicales de cultivo.

Por lo expresado anteriormente, el objetivo del presente trabajo de investigación ha sido evaluar y comparar los componentes ecofisiológicos determinantes del rendimiento de tres especies (mandioca-albahaca-coriandro) cultivadas bajo el sistema consociado en relación a sus respectivos monocultivos.

\section{MATERIALES Y MÉTODOS}

El ensayo se llevó a cabo en el Campo Didáctico Experimental de la Facultad de Ciencias Agrarias ( $\left.27^{\circ} 28^{\prime} 27^{\prime} \mathrm{S}, 58^{\circ} 47^{\prime} 00^{\prime} \mathrm{O}\right)$ de la Universidad Nacional del Nordeste (UNNE), ubicado al noroeste de la Provincia de Corrientes, Argentina.

El suelo del sitio de experimentación ha sido clasificado como Udipsammentes árgico, mixta, hipertérmica, perteneciente a la serie Ensenada Grande (Escobar et al., 1994).

El clima de la región según Köppen corresponde a Cf w* a (h), mesotermal, cálido templado, sin estación seca con precipitaciones máximas en otoño y veranos muy cálidos (Pascale y Damario, 2004). La temperatura media anual varía alrededor de $21,5^{\circ} \mathrm{C}$, la temperatura media del mes más frío (julio) entre 16 y $13^{\circ} \mathrm{C}$ y la media del mes más cálido (enero) entre 27 y $26^{\circ} \mathrm{C}$. En verano se registran máxima absolutas de 42,5 a $46,5^{\circ} \mathrm{C}$, según zonas, y en invierno, mínimas absolutas de -1 a $-5^{\circ} \mathrm{C}$. Sin embargo, las heladas son poco frecuentes, con 320 a 360 días libres de heladas, con frecuencias medias anuales de 0,4 (junio-julio), no registrándose ninguna entre octubre y abril. Las lluvias abundantes y frecuentes, superan $1500 \mathrm{~mm}$ anuales, con distribución irregular, siendo el otoño la época más lluviosa y el invierno la más seca, con un máximo secundario en primavera y un mínimo secundario en verano. Por la precipitación registrada no se puede hablar de estaciones secas ni de sequías de importancia.

La experiencia dio inicio el 28 de septiembre de 2011 con la preparación del suelo con rastra de disco y posterior pasada de rastrillo. Luego se procedió a la marcación del terreno para establecer los experimentos en un diseño de bloques completos al azar, con 4 tratamientos y 3 repeticiones para cada uno de ellos. Los tratamientos fueron los siguientes: T1: cultivo consociado mandioca-albahaca-coriandro, T2: monocultivo de mandioca cv. Rocha, T3: monocultivo de albahaca var "Genovesa" T4: monocultivo de coriandro tipo marroquí. 
El cultivo de mandioca se implantó en octubre, utilizando estacas de $10 \mathrm{~cm}$ de longitud en posición horizontal y con una densidad de 10.000 plantas ha', a $1 \mathrm{~m} \times 1 \mathrm{~m}$ tanto bajo sistema de monocultivo como en asociación con las especies condimentarias que se implantaron sucesivamente a lo largo del ciclo del cultivo principal (octubre-julio) al tiempo que se establecían los monocultivos de las mismas. Las parcelas que contaban con mandioca ( $\mathrm{T} 1$ y T2) presentaban cinco lineos de los cuales se utilizaron los tres centrales para la toma de datos. Las parcelas del tratamiento consociado (T1) estaban constituidas por dos lineos de albahaca trasplantados entre los lineos de mandioca, cuyo ciclo se desarrolló entre los meses de octubre a marzo. Una vez cosechada la albahaca se sembraron dos lineos de coriandro entre los lineos de mandioca, cuyo ciclo se extendió entre el mes de mayo y octubre. En ambos cultivos condimentarios la distancia entre lineos contiguos de la misma especie era de $0,50 \mathrm{~m}$ y entre estas y el lineo de mandioca era de $0,25 \mathrm{~m}$.

Los tamaños de las parcelas de monocultivo fueron de $30 \mathrm{~m}^{2}$ en albahaca (T3) y en coriandro (T4), y de $75 \mathrm{~m}^{2}$ en mandioca (T2). Las parcelas de cultivos asociados constaban de $75 \mathrm{~m}^{2}$ (T1).

Previo a la plantación, las estacas de mandioca se trataron con una solución de 80 g de Oxicloruro de Cobre y $30 \mathrm{~cm}^{3}$ de Dimetoato en $20 \mathrm{I}$ de agua, solución en la que fueron inmersas durante 60 minutos.

La albahaca se sembró en septiembre, en speedlings con celdas de $100 \mathrm{cc}$ en las que se utilizó suelo del mismo sitio de experimentación previamente desinfectado con agua a $80^{\circ} \mathrm{C}$. Los speedlings se mantuvieron bajo invernáculo de 100 micrones y con riego por pulverización manual. Antes del trasplante al terreno definitivo, en el mes de octubre, fueron tratados con un fertilizante orgánico mineral (INICIUM®) a razón de 6 ppm y de esta solución se aplicaron $5 \mathrm{~cm}^{3}$ por celda de $100 \mathrm{cc}$.

En el mes de noviembre se instalaron cintas de riego por goteo para suplementar los momentos de déficit hídrico por falta de precipitaciones y se realizó una fertilización a la albahaca con urea granulada $(46 \% \mathrm{~N})$ a razón de $75 \mathrm{~kg} \mathrm{ha}^{-1}$; la mandioca se fertilizó en el mes de diciembre a los 56 días de la plantación con urea granulada (46\% N) a razón de $75 \mathrm{~kg} \mathrm{ha}^{-1}$.

El cultivo de coriandro, se sembró en el mes de mayo directamente en el campo a chorrillo continuo y luego se raleó dejando las plantas más vigorosas y se fertilizó con $100 \mathrm{~kg} \mathrm{ha}^{-1}$ de fosfato diamónico (18-46-0) a la siembra y $75 \mathrm{~kg} \mathrm{ha}^{-1}$ de urea $(46 \% \mathrm{~N})$ a los 60 días de la primera.

Para cada uno de los cultivos se midieron variables relacionadas con los componentes ecofisiológicos del rendimiento y fueron las siguientes:

a-En mandioca (M) fueron: i) Peso fresco de raíces reservantes (PFR), ii) Peso seco de tallos (PST), iii) Peso seco de hojas (PSH), iv) Biomasa seca aérea (BSA), v) Porcentaje de Materia Seca de Raíces (MSR) y vi) Altura de Plantas (AP). Las variables biométricas fueron medidas 270 días después de la plantación (ddp) en el mes de julio, a partir de datos del promedio de 5 plantas $\left(\mathrm{g} \mathrm{pl}^{-1}\right)$, de cada repetición del $\mathrm{T} 1 \mathrm{y}$ del $\mathrm{T} 2 \mathrm{y}$ se estimaron los valores por hectárea $\left(\mathrm{Kg} \mathrm{ha}^{-1}\right)$. La altura promedio de plantas se evaluó 120 días después de la plantación. EI Porcentaje de Materia Seca de Raíces (MSR), se calculó por el método de la gravedad específica (Toro y Cañas, 1983). 
b-En las especies condimentarias albahaca (A) y coriandro (C) los componentes del rendimiento se evaluaron a través de muestreos de seis plantas por repetición extraídas de manera sistemática al azar.

Las plantas de coriandro, se muestrearon cada 15 días desde la emergencia y se realizó el último muestreo en el mes de octubre. En albahaca, solo se realizó un muestreo destructivo en el mes de diciembre. En ambas especies, las plantas fueron seccionadas y los órganos individuales de cada planta secados en estufas a $70^{\circ} \mathrm{C}$ hasta peso constante para ser luego pesados separadamente y obtener el dato de partición de asimilados en ambos sistemas de cultivo para cada especie. Los resultados se expresaron en g MS particionada planta ${ }^{-1}$ a partir de las cuales se estimaron valores por hectárea $\left(\mathrm{Kg} \mathrm{ha}^{-1}\right)$.

En albahaca (A) se midió: i) Peso de la materia fresca de plantas (PFP) ii) Peso de la materia seca de plantas (PSP), iii) Peso de la materia fresca de inflorescencias (PFI) iv) Peso de la materia seca de inflorescencia (PSI), v) Peso de la materia fresca de tallos (PFT), vi) Peso de la materia seca de tallos (PST),vii) Peso de la materia seca de hojas (PSH) y viii) Peso de la materia fresca de hojas (PFH).

En coriandro (C) se midió: i) Número de Granos por Planta (NGPP), ii) Número de Umbelas por Planta (NUPP), iii) Número de Ramificaciones por Planta (NRPP), iv) Peso Promedio de Mil Granos (PPMG), v) Peso de Granos por Planta (PGPP)y vi) Rendimiento de Granos $\left(\mathrm{kg} \mathrm{ha}^{-1}\right)$.

Los datos registrados en el campo fueron evaluados estadísticamente a través de la prueba de $\mathrm{T}$ con muestras no pareadas a dos colas $(\alpha 0,05)$ para lo que se utilizó el software InfoStat (2002). Los resultados muestran el comportamiento de cada cultivo en consociación versus su monocultivo.

Para la evaluación biológica se tomó el concepto del Uso Equivalente Tierra (UET), que es la suma de dos o más cocientes, según el número de cultivos que intervengan en la asociación, en donde cada cociente representa la relación entre la producción obtenida en la asociación y en el monocultivo, para cada una de las especies. La sumatoria de estos cocientes indica el UET del sistema, que representa la superficie relativa cultivada en monocultivo necesaria para obtener la misma producción que en la asociación:

$$
\mathrm{UET}=\mathrm{Ix}+\mathrm{Iy}+\mathrm{Iz} \ldots=\mathrm{Ax} / \mathrm{Mx}+\mathrm{Ay} / \mathrm{My}+\mathrm{Az} / \mathrm{Mz} \ldots
$$

donde los Ax, Ay, y Az representan los rendimientos obtenidos en la asociación y Mx, My y Mz son los rendimientos de las especies en monocultivo (Mead y Willey, 1980; Mattos y Souza, 2005).

\section{RESULTADOS Y DISCUSIÓN}

Al analizar estadísticamente los resultados a través del estadístico T de Students $(\mathrm{p} \leq 0,05)$, se observaron diferencias significativas entre las medias de los tratamientos del cultivo de mandioca (Cuadro 1) en monocultivo (T2) y consociado (T1) en relación a todas las variables relacionadas a la biomasa aérea (BSA) y su partición a los tallos (PST) y hojas (PSH), tanto como en lo relativo al rendimiento expresado como peso fresco de raíces (PFR). 
Cuadro 1: Efectos del tratamiento en monocultivo (T2) y en consociación (T1) en mandioca (M) sobre las variables asociadas al rendimiento $\left(\mathrm{kg} \mathrm{ha}^{-1}\right)$ en términos de Peso Fresco de Raíces (PFR). Biomasa Seca Aérea (BSA) y su partición de asimilados en Peso Seco de Tallos (PST) y Hojas (PSH) y el Porcentaje de Materia Seca de raíces (MSR)

\begin{tabular}{cccc}
\hline Variable & Media (T1) & Media (T2) & p valor $(\leq 0,05)$ \\
\hline PFR $\left(\mathrm{kg} \mathrm{ha}^{-1}\right)$ & 5200.00 & 13500,00 & $0,0034^{*}$ \\
BSA $\left(\mathrm{kg} \mathrm{ha}^{-1}\right)$ & 1190,67 & 3120,00 & $0,0024^{*}$ \\
PSH $\left(\mathrm{kg} \mathrm{ha}^{-1}\right)$ & 728,00 & 1904,67 & $0,0042^{*}$ \\
PST $\left(\mathrm{kg} \mathrm{ha}^{-1}\right)$ & 462,67 & 1215,33 & $0,0014^{*}$ \\
MSR $(\%)$ & 29,67 & 32,00 & $0,5393^{\text {ns }}$ \\
\hline
\end{tabular}

Prueba de $\mathrm{T}$ con muestras no pareadas a dos colas $(\alpha 0,05)$

El rendimiento de raíces (PFR) del T1 se encontró por debajo de la media citada para la Provincia de Corrientes que es de $12500 \mathrm{~kg} \mathrm{ha}^{-1}$. En consociación (T1) las variables bajo análisis sufrieron reducciones estadísticamente significativas que se encuentran coincidentemente en $38 \%$ para todos de los valores medios de PFR, BSA, PST y PSH. Estas reducciones de la productividad del cultivo de mandioca bajo el sistema consociado respecto a la misma especie implantada en monocultivo, fueron ampliamente observadas en experiencias llevadas a cabo por diferentes autores (Cenóz et al., 1995; Cenóz y Ferrero, 2002; Mattos y Souza, 2005, Albuquerque et al., 2012). Si bien el sistema consociado presenta grandes ventajas como retornos económicos más rápidos, aumento de la eficiencia productiva, intensificación temporo-espacial de los recursos disponibles como agua, nutrientes y radiación solar; por otro lado no suele indicarse para los cultivos comerciales debido a la caída del rendimiento agrícola, contemplándose la posibilidad de compensar la menor productividad con un mayor precio de los cultivos asociados. La materia seca de raíces de mandioca fue la única variable que no manifestó diferencias estadísticas entre tratamientos y se mantuvo dentro de los valores normales que varían entre 20 y $45 \%$ (Toro y Cañas, 1983). En este sentido, similar comportamiento de la materia seca fue citada por Albuquerque et al. (2012), quienes evaluaron la consociación mandioca-Phaseolus vulgaris versus sus respectivos monocultivos. Sin embrago mientras Albuquerque et al. (2012), no encontraron diferencias en la biomasa aérea, en este trabajo las reducciones significativas de la BSA y específicamente de la BSH son desde el punto de vista ecofisiológico la causa que permitiría explicar las reducciones del PFR, producto de la alteración de la relación fuente-destino.

Al analizar la altura de las plantas de albahaca y de mandioca (Cuadro 2) dentro de lo que se considera la ventana crítica de competencia del cultivo de mandioca $(0-150$ días después de la plantación) se observó que en consociación (T1) ambas especies alcanzaban alturas similares, $0,38 \mathrm{~m}$ y $0,42 \mathrm{~m}$ respectivamente. Es probable que esta condición de competencia haya sido lo que repercutió negativamente en la biomasa total y finalmente en el rendimiento del cultivo de mandioca en consociación (Cuadro 1). Olasantan et al. (1995) estudiando el crecimiento y rendimiento de la mandioca consociada con maíz, observaron que el stress causado por la competencia temprana entre las plantas, genera detrimentos irreversibles en el rendimiento de raíces de mandioca. Estos tiempos se asimilarían al período crítico de competencia con malezas comprendido entre los 6090 días después del plantación (Odair \& Contiero, 2006). La reducción de los rendimien- 
tos posiblemente ocurrió como consecuencia del sombreamiento generado por la albahaca, que disminuyó la capacidad de intercepción de la radiación solar del cultivo de mandioca (Olasantan et al., 2012), lo que se suma a una menor BSH. En términos generales, según lo postulado por Mojena y Bertolí (2004), quienes también estudiaron los efectos de la mandioca intercalada con maíz; la magnitud de la competencia entre cultivos consociados depende fundamentalmente del arreglo entre hileras y la densidad de siembra que se utilice, que en este ensayo fue de lineos dobles de albahaca en el entrelineo de mandioca.

Cuadro 2: Alturas promedio de plantas de mandioca (T2) y albahaca (T3) en monocultivo y de ambas especies en consociación (T1) medidas a los $120 \mathrm{ddp}$.

\begin{tabular}{cc}
\hline Tratamiento & Altura promedio (m) \\
\hline Albahaca (T1) & 0,38 \\
Mandioca (T1) & 0,42 \\
Mandioca (T2) & 0,53 \\
Albahaca (T3) & 0,49 \\
\hline
\end{tabular}

Cuadro 3: Medias obtenidas para el cultivo de albahaca (A) para los tratamientos del monocultivo (T3) y en consociación (T1) para las variables: Peso Fresco (PFP) y Seco de Plantas (PSP), Peso Fresco (PFI) y Seco de Inflorescencia (PSI), Peso Fresco (PFT) y Seco de Tallos (PST), Peso Seco (PSH) y Fresco de Hojas (PFH) en kg ha' ${ }^{-1}$.

\begin{tabular}{lccc}
\hline Variable & Media $(\mathrm{T} 1)$ & Media $(\mathrm{T} 3)$ & $\mathrm{p}$ valor $(\leq 0,05)$ \\
\hline PFPL $\left(\mathrm{kg} \mathrm{ha}^{-1}\right)$ & 6583,33 & 6588,89 & $0,9972^{\mathrm{ns}}$ \\
PSPL $\left(\mathrm{kg} \mathrm{ha}^{-1}\right)$ & 944,44 & 1177,78 & $0,2774^{\mathrm{ns}}$ \\
PFH $\left(\mathrm{kg} \mathrm{ha}^{-1}\right)$ & 2527,78 & 3005,56 & $0,5074^{\mathrm{ns}}$ \\
PSH $\left(\mathrm{kg} \mathrm{ha}^{-1}\right)$ & 466,67 & 538,89 & $0,3814^{\mathrm{ns}}$ \\
PFIN $\left(\mathrm{kg} \mathrm{ha}^{-1}\right)$ & 164,00 & 273,33 & $0,1872^{\mathrm{ns}}$ \\
PSIN $\left(\mathrm{kg} \mathrm{ha}^{-1}\right)$ & 200,00 & 333,33 & $0,1872^{\mathrm{ns}}$ \\
PST $\left(\mathrm{kg} \mathrm{ha}^{-1}\right)$ & 1772,22 & 1719,44 & $0,8745^{\mathrm{ns}}$ \\
PFT $\left(\mathrm{kg} \mathrm{ha}^{-1}\right)$ & 2286,17 & 2218,08 & $0,8745^{\mathrm{n}}$ \\
\hline
\end{tabular}

Prueba de $\mathrm{T}$ con muestras no pareadas a dos colas $(\alpha 0,05)$

En el cultivo de albahaca por su parte (Cuadro 3), no se observaron diferencias significativas para las variables medidas entre los tratamientos monocultivo (T3) y consociado (T1), pero estos fueron muy inferiores a los $10000 \mathrm{~kg} \mathrm{ha}^{-1}$ que cita Muñoz (1993) para España, a los $12013 \mathrm{~kg} \mathrm{ha}^{-1}$ de material fresco en el primer corte citados por Curioni y Arizio (1997) en la provincia de San Luis, Argentina y a los de 12.000 a $18.000 \mathrm{~kg} \mathrm{ha}^{-1}$. en Corrientes, Argentina que citan Cenóz et al. (2005) en plantas que se cosechan entre los 90 y 110 días después de plantadas. Los bajos rendimientos obtenidos en este ensayo se pueden explicar desde tres puntos clave del manejo: i) el cultivo de albahaca se cosechó muy tempranamente (60 después del trasplante) antes del momento óptimo de cosecha para alcanzar el máximo rendimiento potencial citado, ii) recién al mes de trasplantadas (noviembre) se suplementaron los déficits hídricos mediante goteo superficial que retrasó la recuperación del stress postrasplante, y finalmente iii) la fertilización nitrogenada, pudo haber sido lixiviada por el mismo riego suplementario. 
Cuadro 4: Medias obtenidas para los tratamientos en monocultivo (T4) y consociación (T1) de coriandro para las variables asociadas al rendimiento en términos de Número de Granos por Planta (NGPP). Número de Umbelas por Planta (NUPP), Número de Ramificaciones por Planta (NRPP), Peso (g) Promedio de los Mil Granos (PPMG), Peso (g) de Granos por Planta (PGPP) y el rendimiento (RG) en kg ha ${ }^{-1}$.

\begin{tabular}{lccc}
\hline \multicolumn{1}{c}{ Variable } & Media $(\mathrm{T} 1)$ & Media $(\mathrm{T} 4)$ & $\mathrm{p}$ valor $(\leq 0,05)$ \\
\hline NGPP & 79,08 & 72,08 & $0,324^{\mathrm{ns}}$ \\
NUPP & 7,42 & 5,67 & $0,041^{*}$ \\
PGPP $(\mathrm{gr})$ & 2,34 & 1,40 & $0,003^{*}$ \\
NRPP & 8,42 & 6,67 & $0,137^{\text {ns }}$ \\
PPMG $(\mathrm{gr})$ & 21,26 & 12,74 & $0,003^{*}$ \\
RG $\left(\mathrm{kg} \mathrm{ha}^{-1}\right)$ & 935,33 & 560,67 & $0,003^{*}$ \\
\hline
\end{tabular}

Prueba de $\mathrm{T}$ con muestras no pareadas a dos colas $(\alpha, 0,05)$

En relación al coriandro (Cuadro 4), se observó que la consociación (T1) favoreció estadísticamente el NUPP, el PGPP, el PPMG y el RG. Según De la Fuente et al. (2006), el NUPP es el componente del rendimiento más variable con relación a las decisiones de manejo, el NGPP explica aproximadamente el $86 \%$ del rendimiento a alcanzar, mientras que PGPP explica aproximadamente el $30 \%$ del rendimiento. En este ensayo se observó que el NGPP se mantuvo estadísticamente igual en T1 que en T4, pero en T1 el RG fue estadísticamente superior mediado por el componente PGPP. EI NUPP y el NGPP alcanzados en este experimento se corresponden con valores más bajos citados en la bibliografía (De la Fuente et al., 2006) pero el PGPP se corresponde con los valores más altos (De la Fuente et al., 2006; Resende et al., 2010; Thayamini y Brintha, 2010). El NGPP se determina durante las primeras etapas, y en el período final del ciclo, se produce el llenado de granos y se determina el PGPP (De la Fuente et al., 2006), en este trabajo quedó evidenciado la capacidad de compensación de fotoasimilados bajo el sistema consociado, lo cual generó rendimientos aceptables en este sistema de cultivo respecto del monocultivo.

Asimismo, se pudo demostrar la factibilidad del cultivo de coriandro en Corrientes, basado en los rendimientos obtenidos de $935,33 \mathrm{~kg} \mathrm{ha}^{-1}$ bajo sistema consociado (Cuadro 4), lo que es comparable a aquellos citados por los soviéticos y países asiáticos que apenas superan los $1000 \mathrm{~kg} \mathrm{ha}^{-1}$, mientras que los españoles citan rindes entre $1000 \mathrm{y}$ $1500 \mathrm{~kg} \mathrm{ha}^{-1}$. Asimismo, el rendimiento promedio a nivel nacional es de $1000-1200 \mathrm{~kg}$ $\mathrm{ha}^{-1}$; que en ciertos casos baja a 700-900 $\mathrm{kg} \mathrm{ha}^{-1}$ (Curioni y Arizio, 1997). La densidad óptima económica de plantas recomendada ronda las 120 a 200 plantas $\mathrm{m}^{2}$ (De la Fuente et al., 2006), y en este ensayo se trabajó con un promedio de 160 plantas $\mathrm{m}^{2}$ con los que se alcanzó el rendimiento mencionado. Los resultados encontrados en este experimento demuestran que el coriandro presenta potencial como especie de renta, y que se desarrolla cuando la mandioca se encuentra en fase de reposo, siendo así una oportunidad de uso del suelo y fuente de trabajo para la comunidad en épocas donde escasea el nivel ocupacional. 
Cuadro 5: Comparación del Uso Equivalente Tierra (UET) obtenido para los diferentes cultivos evaluados en sistemas consociados respecto a cada monocultivo.

\begin{tabular}{lc}
\hline \multicolumn{1}{c}{ Cultivo } & Uso eficiente de la tierra \\
\hline Mandioca & 0,38 \\
Albahaca & 0,99 \\
Coriandro & 1,66 \\
Total & 3,03 \\
\hline
\end{tabular}

Como indicador útil de intensificación del sistema, en el Cuadro 5, se puede observar el Índice del Uso Equivalente de la Tierra (UET) calculado para cada uno de los tratamientos, habiéndose obtenido el máximo aprovechamiento $(3,03)$ con la asociación.

$\mathrm{UET}=\mathrm{Ix}+\mathrm{Iy}+\mathrm{Iz} \ldots=\mathrm{Ax} / \mathrm{Mx}+\mathrm{Ay} / \mathrm{My}+\mathrm{Az} / \mathrm{Mz} \ldots$

donde los Ax, Ay, y Az representan los rendimientos obtenidos en la asociación y Mx, My y Mz son los rendimientos de las especies en monocultivo.

$\mathrm{UET}=\mathrm{Ax} / \mathrm{Mx}+\mathrm{Ay} / \mathrm{My}+\mathrm{Az} / \mathrm{Mz}=$

UET $=935,33 / 560,67+6583 / 6588,89+5200 / 13500=$

UET $=1,66+0,99+0,38$

$\mathrm{UET}=3,03$

De acuerdo a lo expresado, se comprueba la eficiencia del sistema consociado (T1), ya que para obtener los mismos rendimientos bajo sistema de monocultivo se necesitarían 3,03 ha $(0,38$ ha para el cultivo de mandioca, 1 ha para albahaca y 1,66 ha para el cultivo de coriandro).

Asimismo, se demuestra que dado que se logra producir tres cultivos por año en el mismo espacio, el Índice de Intensificación de la secuencia es de 3 con una mayor biomasa producida por unidad de recurso capturado.

A juzgar por los resultados hallados, la convivencia temprana con otro cultivo (albahaca) afecta negativamente el cultivo de mandioca causando efectos depresivos en las variables biométricas y particularmente sobre los rendimientos de raíces. Asimismo, se podría considerar realizar el trasplante más tardío para la albahaca, cuando sea mayor la altura del cultivo de mandioca, a fin de minimizar la competencia interespecífica por el recurso luz, o bien variar el diseño espacial de la asociación, colocando solo un lineo de albahaca en el entresurco de mandioca para maximizar la complementación entre especies. La consociación al final del ciclo del cultivo de mandioca, sería altamente recomendable, no afectaría los rendimientos y el coriandro presenta aptitud en términos de rendimiento.

De esta manera, con los resultados preliminares obtenidos a partir de este ensayo experimental exploratorio, quedaría demostrado el eficiente uso del espacio de estas consociaciones, y se demostraría que es recomendable su aplicación en esquemas de agriculturas familiares, logrando diversificar la producción y redituando en beneficios económicos totales. Para validar los resultados definitivos, el experimento deberá repetirse bajo idénticas condiciones y también sería recomendable realizar otros nuevos ensayos para poder evaluar la consociación de la mandioca con la albahaca, reprogramando la distri- 
bución espacial y temporal, para eficientizar el sistema y reducir pérdidas en el rendimiento de raíces del cultivo principal.

\section{Conclusiones}

1-La consociación de mandioca con especies condimentarias como albahaca y coriandro es factible agronómicamente y en términos de uso eficiente de la tierra cuyo valor alcanza 3 .

2-La disminuciones del rendimiento biológico de la mandioca en el sistema consociado, se explican por la alta competencia interespecífica temprana ejercida por la albahaca, durante un período que se considera crítico, que genera reducciones en la intercepción de la alta demanda inicial de radiación lumínica de la mandioca y en la biomasa aérea cuyo efecto negativo no se revierte y consecuentemente repercute en el detrimento del rendimiento final de raíces.

3-La biomasa particionada y los rendimientos de la albahaca en consociación no se diferencian estadísticamente del monocultivo.

4-El coriandro consociado con mandioca, presenta rendimientos superiores que se explican por un mayor peso de granos, lo que resulta altamente recomendable en términos de UET y de manejo de un sistema sustentable.

\section{AGRADECIMIENTOS}

A la Secretaría General de Ciencia y Técnica del Universidad Nacional del Nordeste UNNE (SGCyT-UNNE) por el financiamiento del PI A006/10 y PI A002/14.

\section{REFERENCIAS}

Albuquerque, J.A.A.; S. Tocio, J.M. Arcanto Al.ves; A.A. DA Silva y S.C. Phirbira UchoA. 2012. Cultivo de mandioca e feijão em sistemas consorciados realizado em Coimbra, Minas Gerais, Brasil. Ciencia Agronómica, v.43, p.532-538.

Alvfs, A.A. 2002. Cassava Botany and Physiology. In: Hillocks, R.J., J.M. Tres and A.C. Bellotti (Eds.): Cassava: Biology, Production and Utilization. CAB International. London, U.K. 352 p.

Alves de MendoçA, H.; G.M. DE Melo y E.C. TeixeIRA, 2003. Avaliação de genotipos de mandioca em diferentes épocas de colheita no Estado do Acre. Pesquisa Agropecuária Brasileira, v. 38, p.761769.

Cenóz, P.J.; A.M. Burgos y A.E. LôPEz, 2005. Influencia de la Fertilización Nitrogenada en el Rendimiento de Albahaca (Ocimm basilicum, L.). Revista Horticultura Argentina, v. 24, p.18-20

Cenóz, P.J. y A.R. Ferrero, 2002. Análisis productivo y alimentario de cultivos asociados. Revista Internacional del Centro de Información Tecnológica (CIT), v. 13, p. 9-14.

Cenóz, P.J.; J. Schroeder y R. Karactnque, 1995. Análisis comparativo del efecto de la densidad y distribución de plantas en una consociación Mandioca-Caupí. Revista Horticultura Argentina, v. 14, p.1216. 
Chnóy, P.J.; R. Karacinquh y J. Schrotilfr, 1998. Efecto de una consociación Mandioca-Batata, en su aspecto cultural y económico. Revista Horticultura Argentina, v. 17, p. 32-35.

CrNóy, P.J.; A.M. Burgos y C.N. BAI.BI, 2010. La densidad de plantas como variable de rendimiento cultural y económico de cuatro cultivos consociados bajo las condiciones agroecológicas del nordeste de $\mathrm{Ar}$ gentina (NEA). Revista Horticultura Argentina, v. 29 , p.18-25.

Curıoni, A. y O. Ari\%ıo, 1997. Plantas Aromáticas y Medicinales. Umbeliferas. Buenos Aires, Argentina. Hemisferio Sur. p. 1-32.

De la Fuente, E.B; A. Gil; P.I. Gimenez; A.G. K^ntoloK; M. Lopez Pereirı y E. Ploschuk, 2006. Cultivos Industriales. Bs As, Ed. FAUBA. 800 p.

Escobar, E.; D. Ligitir; M. Mrigar; H. Mat"l'io y O. Val.l.jos, 1994. Mapa de suelos de los Deptos. Capital, San Cosme e Itati de la Pcia. Corrientes. Convenio INTA-ICA y Pcia. Corrientes-CFI. 129 p.

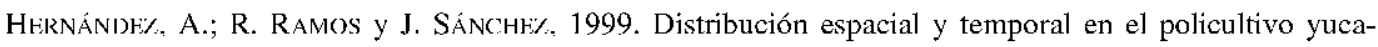
frijol: uso equivalente de la tierra. Agronomia Mesoamericana, v. 10, p. 63-66.

InfoStAT. Grupo InfoStat. 2002. Facultad de Ciencias Agropecuarias, Universidad Nacional de Córdoba, Argentina.

LIGIER, H.D., 1997. Estrategias para una agricultura sustentable en pequeñas propiedades. E.E.A. INTA Corrientes, Argentina. $20 \mathrm{p}$.

MA'Tros, P.L.P. y A.S Sou\%.A, 2005. Consorciação da Mandioca plantada em fileiras duplas e simples com culturas de ciclo curto I. mandioca x caupi x milho. Revista Brasileira de Mandioca. Cruz das Almas, v. 18 , p. $25-30$.

Mral), R. y R. Wıllty, 1980. The concept of a "Land Equivalent Ratio" and advantages in yield from intercropping. Experimental Agriculture, vol. 16, p. 217-228.

Mojens, M. y M. Bertoli. 2004. Rendimiento de la Yuca (Manihot esculentaCrantz) en diferentes arreglos espaciales. Agronomía Costarricense, vol. 28, p. 87-94.

MuÑo\%, F., 1993. Plantas medicinales y aromáticas. Estudio, cultivo y procesado. Ediciones Mundi-Prensa, Madrid.

ODAIR, J. y R.L. CONTIERo, 2006. Efeitos de diferentes períodos de controle e convivência de plantas daninhas com cultura da mandioca. Ciencia Agronómica, v.37, p.326-331.

Olasantan, F.O.; H.C. EzUMA y E.O. LUCAS, 1995. Effects of intercropping with maize on the microenvironment, growth and yield of cassava. Agriculture, Ecosystems and Environment, v. 57, p. 149158.

Pascal.t, A.J. y E.A. Damario, 2004. Bioclimatología Agrícola y Agroclimatología. Editorial Facultad de Agronomía, Universidad de Buenos Aires. 550 p.

Resende, A.S; A.J.; Das Viana; R.J. Oliveira; E. Del Aguiar-Menezes; R. Del Ribeiro; M. Doss FricCl y J.G.M. GujRrRA, 2010. Consórcio couve-coentro em cultivo orgânico e sua influência nas populações de joaninhas. Horticultura Brasileira, v.28: 41-46.

Thay Mmini, H.S. e I. BRintha, 2010. Review on Maize Based Intercropping. Journal of Agronomy, v. 9, p $135-145$

TORO, J.C. y A. CAÑAs, 1983. Determinación del contenido de materia seca y almidón en yuca por el sistema de gravedad específica. Pp. 567-575. In: Domínguez, C. (Ed.); Yuca: Investigación, producción y utilización. Cali, Colombia. 